\title{
Probing linearly polarized gluon distribution in electron-proton collision at EIC
}

\author{
Raj Kishore* \\ Department of Physics, Indian Institute of Technology Bombay \\ Powai, Mumbai 400076, India \\ E-mail: raj.thepsegmail.com
}

\section{Asmita Mukherjee}

Department of Physics, Indian Institute of Technology Bombay

Powai, Mumbai 400076, India

E-mail: asmita@phy.iitb.ac.in

\begin{abstract}
We present a recent estimate [8] of azimuthal asymmetry $(\cos 2 \phi)$ in $J / \psi$ production in ep collision within the kinematics of the planned electron-ion collider (EIC). We find a small but sizable asymmetry in the small- $x$ region which can be observed at the EIC. This asymmetry directly probes the Weizsäcker-Williams (WW) type linearly polarized gluon distribution in the unpolarized proton. We calculate the asymmetry at next-to-leading order in the kinematical region which satisfies, energy fraction of $J / \psi, z<1$ and the subprocess $\gamma^{*}+g \rightarrow c+\bar{c}+g$ gives the leading contribution. We use color singlet (CS) model for $J / \psi$ production. We estimate the asymmetry in two models for TMDs: (i) Gaussian type parameterization and (ii) McLerran-Venugopalan (MV) model at small- $x$ and also we present the upper bound of the asymmetry.
\end{abstract}

Light Cone 2019 - QCD on the light cone: from hadrons to heavy ions - LC2019

16-20 September 2019

Ecole Polytechnique, Palaiseau, France

\footnotetext{
${ }^{*}$ Speaker.
} 


\section{Introduction}

$J / \psi$ electroproduction can be a probing channel for direct detection of linearly polarized gluon transverse momentum dependent parton distribution (TMDs). We don't have much information about gluon TMDs, just that it satisfies positivity bound. Like the quark TMDs, it depends on the process but the process dependence of gluon TMDs is more complicated due to the presence of two gauge links [1]. In the literature, in the small- $x$ physics, when the gauge links are both future pointing $[++]$ or past pointing $[--]$, the TMD is called Weizsäcker-Williams (WW) gluon distribution [2,3]. Gluon TMDs with one future and one past pointing [+-] or $[-+]$ gauge links are called Dipole distribution [4]. Linearly polarized gluon distribution $h_{1}^{\perp g}$ was first introduced in Ref.[5]. It is a time-reversal even function and can be WW type or Dipole distribution depending on the gauge links. It affects the unpolarized cross section and causes azimuthal asymmetry like $\cos (2 \phi)$ [6]. At leading order (LO), it can be probed in the process $\gamma^{*}+g \rightarrow c \bar{c} \rightarrow J / \psi$ contributing at $z=1$ [7], where $z$ is the energy fraction of the photon carried by the $J / \psi$ in the proton rest frame. So far $h_{1}^{\perp g}$ has not been extracted from experiments. We extend the kinematics to $z<1$ where the leading process is $\gamma^{*}+g \rightarrow c \bar{c}+g \rightarrow J / \psi+g$. We use color singlet (CS) model for $J / \psi$ production [8]. We estimate the $\cos (2 \phi)$ asymmetry in two different model parametrization for TMDs namely Gaussian parametrization and McLerran-Venugopalan (MV) model.

\section{Framework for calculation}

The process we consider is

$$
e(l)+p(P) \rightarrow e\left(l^{\prime}\right)+J / \psi\left(P_{h}\right)+X
$$

where both the electron and proton are unpolarized. The four momentum of the incoming and outgoing particles are given in round brackets. We consider virtual photon-proton center of mass frame. For the small- $x$, the dominating subprocess is virtual photon-gluon fusion process: $\gamma^{*}(q)+$ $g(k) \rightarrow J / \psi\left(P_{h}\right)+g\left(p_{g}\right)$, this contributes in the kinematic region $z<1$ which can be accessed at the future electron-ion collider (EIC). Here, $z=P \cdot P_{h} / P \cdot q$, is the fraction of energy of $\gamma^{*}$ taken by $J / \psi$ in the proton rest frame. We consider the frame in which the virtual photon and proton are in $+z$ and $-z$ directions respectively. We define a lepton plane, formed by the incoming and outgoing electron, for measuring angles of other particles. Within the generalized factorization scheme with the inclusion of intrinsic transverse momentum, the differential cross section for the unpolarized process is given by [8]

$$
\begin{aligned}
d \sigma= & \frac{1}{2 s} \frac{d^{3} l^{\prime}}{(2 \pi)^{3} 2 E_{l^{\prime}}} \frac{d^{3} P_{h}}{(2 \pi)^{3} 2 E_{P_{h}}} \int \frac{d^{3} p_{g}}{(2 \pi)^{3} 2 E_{g}} \int d x d^{2} \boldsymbol{k}_{\perp}(2 \pi)^{4} \delta^{4}\left(q+k-P_{h}-p_{g}\right) \\
& \times \frac{1}{Q^{4}} L^{\mu \mu^{\prime}}(l, q) \Phi^{v v^{\prime}}\left(x, \boldsymbol{k}_{\perp}\right) \mathscr{M}_{\mu \nu}^{\gamma^{*}+g \rightarrow J / \psi+g} \mathscr{M}_{\mu^{\prime} v^{\prime}}^{* \gamma^{*}+g \rightarrow J / \psi+g}
\end{aligned}
$$

where $L^{\mu \mu^{\prime}}$ is leptonic tensor and for the unpolarized proton, the gluon correlator, $\Phi^{v v^{\prime}}$ can be parameterized in terms of unpolarized gluon distribution, $f_{1}^{g}\left(x, \boldsymbol{k}_{\perp}^{2}\right)$ and linearly polarized gluon distribution $h_{1}^{\perp g}\left(x, \boldsymbol{k}_{\perp}^{2}\right) . J / \psi$ is produced within color singlet (CS) model. Since, $J / \psi$ is a ${ }^{3} S_{1}$ 
state, in CS model the $c \bar{c}$ pair produced in the partonic scattering process: $\gamma^{*}+g \rightarrow c+\bar{c}+g$ has to be in ${ }^{3} S_{1}$ state and the scattering amplitude is given by [8]

$$
\mathscr{M}\left[{ }^{3} S_{1}^{(1)}\right]\left(P_{h}, k\right)=\frac{1}{4 \sqrt{\pi M}} R_{0}(0) \frac{\delta_{a b}}{\sqrt{N_{c}}} \operatorname{Tr}\left[\mathscr{O}(0)\left(-P_{h}+M\right) \dot{\xi}_{s_{z}}\right],
$$

where, operator $\mathscr{O}(0)$ is calculated from Feynman diagrams contributing to the process [8], is given as

$$
\begin{gathered}
\mathscr{O}(0)=\left[\frac{\gamma_{v}\left(P_{h}-2 q+M\right) \gamma_{\mu}\left(-P_{h}-2 \not p_{g}+M\right) \gamma_{\rho}}{\left(\hat{s}-M^{2}\right)\left(\hat{u}-M^{2}+q^{2}\right)}+\frac{\gamma_{\rho}\left(P_{h}+2 \not p_{g}+M\right) \gamma_{v}\left(-P_{h}+2 \not k+M\right) \gamma_{\mu}}{\left(\hat{s}-M^{2}\right)\left(\hat{t}-M^{2}\right)}\right. \\
\left.+\frac{\gamma_{v}\left(P_{h}-2 \not q+M\right) \gamma_{\rho}\left(-P_{h}+2 k k+M\right) \gamma_{\mu}}{\left(\hat{t}-M^{2}\right)\left(\hat{u}-M^{2}+q^{2}\right)}\right] g_{s}^{2}\left(e e_{c}\right) \varepsilon_{\lambda_{g}}^{\rho *}\left(p_{g}\right)
\end{gathered}
$$

Here, $\varepsilon_{\lambda_{g}}$ and $\varepsilon_{S_{z}}$ are polarization of final gluon and $J / \psi$ respectively. $M$ is mass of $J / \psi . \hat{s}, \hat{t}$ and $\hat{u}$ are Mandelstam variables of partonic process. $R_{0}$ is radial wave function and associated with long distance matrix elements (LDMEs) [9].

\section{Asymmetry calculation}

Since, we are interested in small- $x$ region, we neglect the terms with higher power of $x_{B}$ and also keep the terms up to $\left(\frac{k_{\perp}^{2}}{M_{p}^{2}}\right)$. The final differential cross section then can be given as [8]

$$
\begin{aligned}
& \frac{d \sigma}{d y d x_{B} d z d^{2} \mathbf{P}_{h T}}=\frac{1}{256 \pi^{4}} \frac{1}{x_{B}^{2} s^{3} y^{2} z(1-z)} \int k_{\perp} d k_{\perp} \\
&\left\{\left(A_{0}+A_{1} \cos \phi_{h}\right) f_{1}^{g}\left(x, \mathbf{k}_{\perp}^{2}\right)\right\}+\frac{k_{\perp}^{2}}{M_{p}^{2}}\left\{\left(B_{0}+B_{1} \cos \phi_{h}+B_{2} \cos 2 \phi_{h}\right) h_{1}^{\perp g}\left(x, \mathbf{k}_{\perp}^{2}\right)\right\}
\end{aligned}
$$

The coefficients, $A_{0}, A_{1}, B_{0}, B_{1}$ and $B_{2}$ are given in the appendix of Ref.[8]. $M_{p}$ is mass of proton. $k_{\perp}$ is the magnitude of $\mathbf{k}_{\perp}$. We estimate the $\cos (2 \phi)$ asymmetry, which is defined as

$$
\left\langle\cos \left(2 \phi_{h}\right)\right\rangle=\frac{\int d \phi_{h} \cos \left(2 \phi_{h}\right) d \sigma}{\int d \phi_{h} d \sigma}
$$

where $\phi_{h}$ is azimuthal angle of $J / \psi$ production plane with the lepton plane. We use two parametrizations of the TMDs to estimate the asymmetry: (i) Gaussian parametrization in which the transverse dependent part in both TMDs, $f_{1}^{g}$ and $h_{1}^{\perp g}$, are Gaussian function [10]. It is a most widely used parameterizarion in the literature. (ii) McLerran-Venugopalan model [11], which can be used to calculate the Weizsäcker-Williams (WW) gluon distribution in small- $x$ region. We also estimated the upper bound of the asymmetry by saturating the positivity bound: $\frac{\mathbf{k}_{\perp}^{2}}{2 M_{p}^{2}}\left|h_{1}^{\perp g}\left(x, \mathbf{k}_{\perp}^{2}\right)\right| \leq f_{1}^{g}\left(x, \mathbf{k}_{\perp}^{2}\right)$

\section{Numerical Results}

We have estimated the $\cos (2 \phi)$ asymmetry in electroproduction of $J / \psi$ within the EIC kinematic region. We used the MSTW2008 [9] for collinear pdfs which obey DGLAP evolution. We 
calculate the asymmetry in small- $x$ region where the gluon initiated processes dominate. This kinematical region will be accessed at EIC and can be important in the determination of gluon TMDs including linearly polarized gluon TMD. For both the parametrization, $\cos (2 \phi)$ asymmetry is negative which is consistent with the calculation in [7]. In the CS model as only one state, namely ${ }^{3} S_{1}$, contributes to the $J / \psi$ production, the asymmetry does not depend on the specific choice of LDMEs. However, the unpolarized cross section will depend on the choice of LDMEs.

(a)

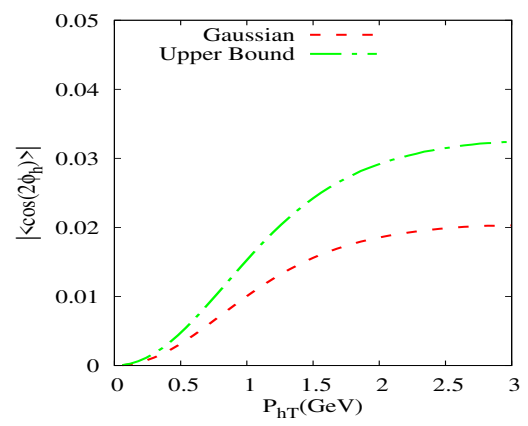

(b)

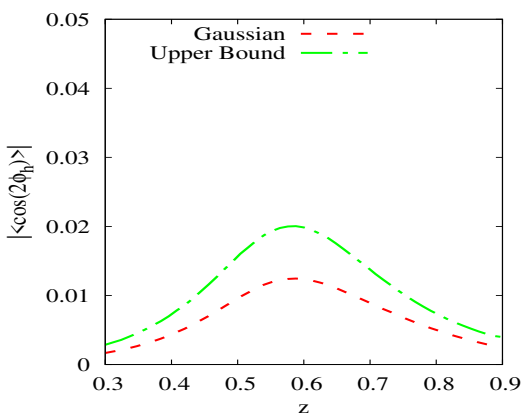

Figure 1: $\cos \left(2 \phi_{h}\right)$ asymmetry in $e+p \rightarrow e+J / \psi+X$ process as function of (a) $P_{h T}$ (left panel) and (b) $z$ (right panel) at $\sqrt{s}=45 \mathrm{GeV}$ (EIC) and $x_{B}=0.01$. [8]

(a)

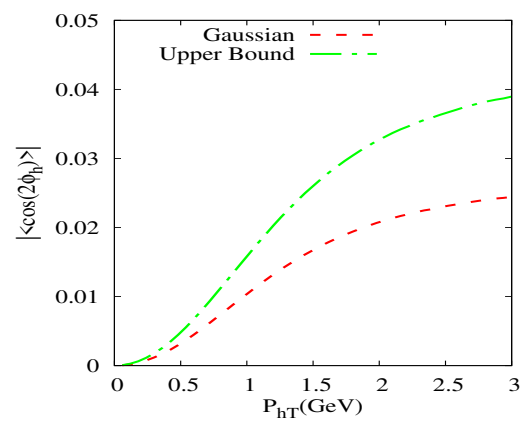

(b)

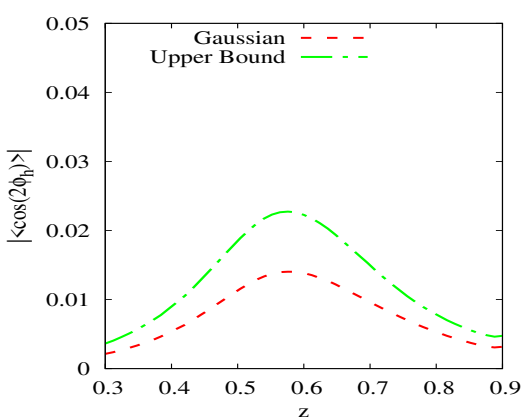

Figure 2: $\cos \left(2 \phi_{h}\right)$ asymmetry in $e+p \rightarrow e+J / \psi+X$ process as function of (a) $P_{h T}$ (left panel) and (b) $z$ (right panel) at $\sqrt{s}=150 \mathrm{GeV}$ (EIC) and $x_{B}=0.01$. [8]

In the figures 1-2, we plotted the upper bound of the asymmetry as well as the magnitude of the asymmetry calculated using Gaussian parametrization, at $\sqrt{s}=45 \mathrm{GeV}$ and $150 \mathrm{GeV}$ respectively as a function of $P_{h T}$ and $z . x_{B}$ is constrained by small- $x$ kinematic region. We took $x_{B}=0.01$. The range of $y$ integration are constrained by the choice of $Q^{2}$ and $x_{B}$. The corresponding range of $y$ integration are $0.05<y<0.4$ and $0.005<y<0.04$ respectively. Transverse momentum $P_{h \perp}$ of the outgoing $J / \psi$ particle is taken in the range $0<P_{h \perp}<3 \mathrm{GeV}$. This upper limit on $P_{h \perp}$ reduces the fragmentation contributions from heavy quark. In all the plots the energy fraction $z$ is in the range $0.3<z<0.9$. The upper bound on $z$ makes the outgoing gluon in partonic process hard and hence there is no contributions from virtual diagrams. Whereas, the lower limit eliminates the fragmentation of hard gluon to $J / \psi$. The upper bound of the asymmetry increases with the increase of centre of mass energy, $\sqrt{s}$, for the fixed $x_{B}$. It reaches a maximum of about $4 \%$ near $P_{h T} \approx 3$ $\mathrm{GeV}$, for $\sqrt{s}=150 \mathrm{GeV}$. The asymmetry reaches a peak near $z=0.6$, but the qualitative behavior remains the same for all $\sqrt{s}$. In Fig. 3, we show a comparison of the upper bound of the asymmetry 
with that calculated in the Gaussian model as well as the MV model. Asymmetry in the MV model is smaller compared to the Gaussian model, and both lie below the upper bound.

(a)

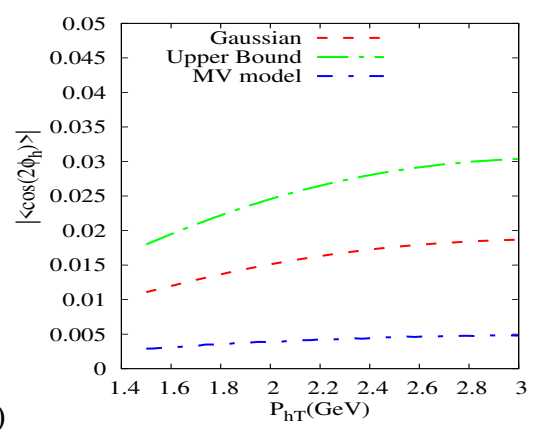

(b)

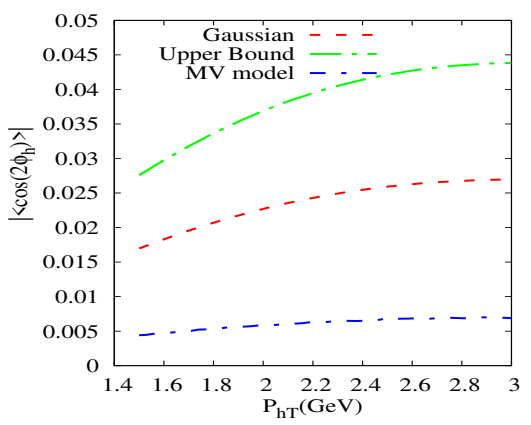

Figure 3: $\cos \left(2 \phi_{h}\right)$ asymmetry in $e+p \rightarrow e+J / \psi+X$ process as a function of $P_{h T}$ for (a) fixed $Q^{2}=$ $9 \mathrm{GeV}^{2}, x=0.01$ and $z=0.5$ and (b) fixed $Q^{2}=9 \mathrm{GeV}^{2}, x=0.01$ and $z=0.7$.[8]

\section{Conclusion}

We estimated the $\cos (2 \phi)$ asymmetry in electroproduction of $J / \psi$ at EIC. We use the CS model to calculate the $J / \psi$ production rate. We found small but a sizable asymmetry that can probe the WW type linearly polarized gluon distribution in the unpolarized proton. The asymmetry is calculated in kinematic region $z<1$ and in small-x, using Gaussian parametrization and MV model for both the TMDs. We also show the upper bound of the asymmetry.

\section{Acknowledgement}

RK would like to thank the organizers of the Light-Cone 2019 conference at Ecole Polytechnique, Palaiseau, France, for the opportunity to present this work and all the support.

\section{References}

[1] M. G. A. Buffing, A. Mukherjee, and P. J. Mulders, Phys. Rev. D88, 054027 (2013), 1306.5897.

[2] Y. V. Kovchegov and A. H. Mueller, Nucl. Phys. B529, 451 (1998), hep-ph/9802440.

[3] L. D. McLerran and R. Venugopalan, Phys. Rev. D59, 094002 (1999), hep-ph/9809427

[4] F. Dominguez, J.-W. Qiu, B.-W. Xiao, and F. Yuan, Phys. Rev. D85, 045003 (2012), 1109.6293.

[5] P. J. Mulders and J. Rodrigues, Phys. Rev. D63, 094021 (2001), hep-ph/0009343.

[6] C. Pisano, D. Boer, S. J. Brodsky, M. G. A. Buffing, and P. J. Mulders, JHEP 10, 024 (2013), 1307.3417ur. Phys. J. C77, 854 (2017), 1609.05596.

[7] A. Mukherjee and S. Rajesh, Eur. Phys. J. C77, 854 (2017), 1609.05596.ur. Phys. J. C77, 854 (2017), 1609.05596 .

[8] R. Kishore and A. Mukherjee, Phys. Rev. D99, 054012 (2019), 1811.07495.

[9] A. D. Martin, W. J. Stirling, R. S. Thorne, and G. Watt, Eur. Phys. J. C 63, 189 (2009).

[10] D. Boer and C. Pisano, Phys. Rev. D86, 094007 (2012), 1208.3642.

[11] L. D. McLerran and R. Venugopalan, Phys. Rev. D49, 2233 (1994), hep-ph/9309289. 